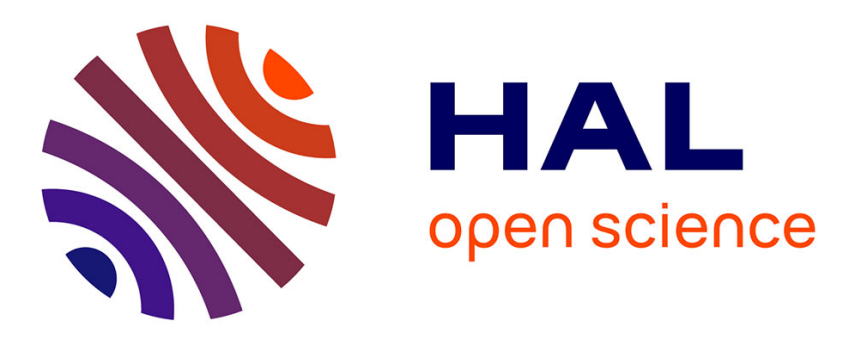

\title{
Agent and Consensus Approaches to Microgrid Coordination for Resilience Improvement
}

Jin Wei, Robin Roche, Abderrafiaa Koukam, Fabrice Lauri

\section{To cite this version:}

Jin Wei, Robin Roche, Abderrafiaa Koukam, Fabrice Lauri. Agent and Consensus Approaches to Microgrid Coordination for Resilience Improvement. International Conference on Management of Digital EcoSystems, Nov 2017, Bangkok, Thailand. hal-02131019

\section{HAL Id: hal-02131019 https://hal.science/hal-02131019}

Submitted on 16 May 2019

HAL is a multi-disciplinary open access archive for the deposit and dissemination of scientific research documents, whether they are published or not. The documents may come from teaching and research institutions in France or abroad, or from public or private research centers.
L'archive ouverte pluridisciplinaire HAL, est destinée au dépôt et à la diffusion de documents scientifiques de niveau recherche, publiés ou non, émanant des établissements d'enseignement et de recherche français ou étrangers, des laboratoires publics ou privés. 


\section{Agent and Consensus Approaches to Microgrid Coordination for Resilience Improvement}

\author{
Jin Wei \\ LE2I \\ Univ. Bourgogne \\ Franche-Comte \\ UTBM, Belfort \\ jin.wei@utbm.fr
}

\author{
Robin Roche \\ FEMTO-ST, CNRS \\ Univ. Bourgogne \\ Franche-Comte \\ UTBM, Belfort \\ robin.roche@utbm.fr \\ Fabrice Lauri \\ LE2I \\ Univ. Bourgogne \\ Franche-Comte \\ UTBM, Belfort \\ fabrice.lauri@utbm.fr
}

\author{
Abderrafiaa Koukam \\ LE2I \\ Univ. Bourgogne \\ Franche-Comte \\ UTBM, Belfort \\ abder.koukam@utbm.fr
}

\begin{abstract}
Microgrids are increasingly popular small-scale power systems with local generation, storage and loads. Interconnecting several microgrids together could provide a flexible structure for power distribution and enable large renewable and distributed penetration levels, as well as coordination between microgrids when disturbances occur. This paper proposes a control strategy for such connected microgrids. Although a microgrid aims to operate independently in normal conditions, several microgrids can support each other in case of contingencies or insufficient generation. Such coordination aims at reducing load shedding and generation curtailment, and is achieved by coordinating the output power of microgrids. Each microgrid is equipped with an agent to achieve self-control and to negotiate with other microgrids, for example to request power to its neighbors to support its loads. The Newton-Raphson and consensus methods are used to calculate the output power of each microgrid. The control strategy is validated using simulations on an IEEE 13-node test feeder.
\end{abstract}

\section{Categories and Subject Descriptors}

C.2.4 [Computer Systems Organization]: Computercommunication NetworksDistributed Systems; B.4.3 [Hardware]: Input/Output and Data Communication-Interconnections (Subsystems)

\section{Keywords}

micro grid, multi-agent system, distributed system, consensus theorem

Permission to make digital or hard copies of all or part of this work for personal or classroom use is granted without fee provided that copies are not made or distributed for profit or commercial advantage and that copies bear this notice and the full citation on the first page. To copy otherwise, to republish, to post on servers or to redistribute to lists, requires prior specific permission and/or a fee.

Copyright 20XX ACM X-XXXXX-XX-X/XX/XX ...\$10.00.

\section{Nomenclature}

$A, B, C$ Parameters of electricity price calculation

$i, j, h$ Indices for MGs

$k \quad$ Iteration step

$p^{c a p, \min }, p^{c a p, \max }$ Power flexibility of MG: min./max. limits

$p_{\text {cap }} \quad$ Line capacity

$p_{E S S, d c h}^{\max }, p_{E S S, c h}^{\max }$ Maximal discharging/charging power of energy storage system

$r, b, x$ Resistance, susceptance, and reactance of a power line

$\delta_{i} \quad$ Voltage angle of MG $i$

$\pi_{i, j}, p_{i, j}$ Price/amount of electricity that MG $i$ sells to MG $j$

a Element of parameter array for consensus algorithm

$l_{i j} \quad$ Set of power lines connecting MGs $i$ and $j$

$M_{i} \quad$ Set of neighbor MGs for MG $i$

$p^{\text {dem }} \quad$ Power demand of MG for the network

$p^{e q u} \quad$ Equivalent load of power line loss connecting MGs

$p^{\text {flow }} \quad$ Power flowing on the power line connecting MGs

$p^{\text {loss }} \quad$ Power loss on the power line connecting MGs

$p^{\text {mod }} \quad$ Equivalent exchanged power of MG with network

$p_{i} \quad$ Power exchanged with the network of MG $i$

$p_{l_{i j}} \quad$ Power flowing on the line connecting MGs $i$ and $j$

pload Load power

$p_{M T} \quad$ Microturbine output

$p_{P V} \quad$ Photovoltaic generator output

$T \quad$ Set of all MGs in the network

$Y \quad$ Electricity price MG sells to its neighbors 


\section{INTRODUCTION}

The smart grid concept has been introduced over the past few years with multiple objectives. One of them is to enable a higher resilience than conventional power systems through high intelligence and automation. Among the various possibilities to achieve this, microgrids (MGs) are small-scale electric systems which can operate independently and connect to the local main electrical grid [Katiraei, Iravani, and LehnKatiraei et al.2005], while equipped with local generation, storage and loads. They are typically designed to possess high reliability and flexibility when faults occur [Roche, Blunier, Miraoui, Hilaire, and KoukamRoche et al.2010]. This can for example improve the immunity of the system to faults occuring on the utility distribution system, typically resulting from bad weather [Panteli, Trakas, Mancarella, and HatziargyriouPanteli et al.2016].

However, as the capability of a single MG is limited, multiple MGs connected together can help to further increase the system resilience, by enabling forms of rescue, e.g., when neighboring MGs exchange power in case one needs it. Additionally, such exchanges can help increase MG profit through sold power. For example, an MG suffering from a failure in its storage system can import power from its neighbors to avoid having to shed load. Coordination between MGs is therefore required.

Control within an MG and coordination with the main electric grid or other MGs are current hot topics in MG research. Several challenges, such as coordination among MGs, hinder the development of MGs and have been little studied so far.

As each MG is independent and the total number of the MGs can be large, information flow is an important concern that affects control strategies. Obtaining global information, such as the output power for all MGs in the network, requires high speed communication. As MGs exchanging power with the network influences the power flow on lines, line capacity should also not be exceeded. Additionally, there is a profit expectation for system operation. To achieve coordination with the above constraints, Nunna and Doolla [Nunna and DoollaNunna and Doolla2012] designed a coordination network control system for achieving supply-demand balance within an MG and the whole network. The coordination of MGs is done by power auctions. Another control strategy was proposed by Liu [Liu, Gao, Liu, Ma, Chen, and YangLiu et al.2015]. It is based on a MAS for MG coordination. However, the power flow among MGs only considers power demand, and the power scheduling for coordination among MGs does not consider network losses. Paper [Wang, Chen, Wang, and ChenWang et al.2016] proposes a radial MG network energy management approach where each MG is controlled by an agent. They communicate with other MGs to determine their output power. Power is exchanged via a common point in the network to achieve coordination. Wang and Chen [Wang, Chen, Wang, Begovic, and ChenWang et al.2015] consider distributed generation and load consumption for each MG and have MGs cooperate via the distribution network operator for minimizing the operation costs. In Marvasti and Fu's work [Marvasti, Fu, DorMohammadi, and Rais-RohaniMarvasti et al.2014], the distributed system and multiple MGs are treated as independent systems. Thus a hierarchical optimization framework is applied to obtain a maximal benefit for both the system and individual MGs. To maxi- mize the profit of the cooperating MGs, Wu and Guan [Wu and GuanWu and Guan2013] solve the economic operation problems with a dynamic programming algorithm, and a decentralized partially-observable Markov decision process is applied for the optimal modeling of the control problem. Paper [Asimakopoulou, Dimeas, and HatziargyriouAsimakopoulou et al.2013] manages the energy transmitted inside an MG network with a leader-follower model and a bilevel program. Considering probabilistic demand, Fathi and Bevrani [Fathi and BevraniFathi and Bevrani2013] schedule the consumption in an MG network with an on-line stochastic algorithm.

The above literature review shows that the topic is timely, and that although centralized approaches have been discussed in a number of studies, decentralized approaches have not yet been explored thoroughly. Such approaches have the advantage of not being subject to single points of failure, of using decision-making as close as possible to the end-users, here the MGs, and of being potentially scalable to large systems. However, decentralized coordination requires solving several challenges, such as: how much power should an MG output to its neighbors when the neighbors do not have enough local energy to supply its own loads? how to distribute the MG output when it has 2 or more neighbor MGs which need energy? how to control each MG's power exchange with its neighbors to reach both global and individual supply-demand balance, while maximizing its economic profit?

In this work, a control strategy for MG network coordination based on MAS is proposed. In this strategy, each MG is controlled by an agent, i.e., an entity capable of taking decisions through interactions with other agents in its environment. In normal operating conditions, agents control resources inside MGs to supply their own load and operate independently from the network. When an MG suffers from a disturbance (e.g., it lacks power because of a component failure), the corresponding agent requests power to its neighbors. To minimize the total operation cost in the network related to handling the disturbance, the power dispatch among MGs is calculated by the requesting agent with the Newton-Raphson method. A consensus algorithm is then adopted for the agents to determine the exchanged power between MGs in the network. With this information, the scheduled power flow in the system is determined, and used to check whether line capacity constraints are satisfied.

The rest of this paper is organised as follows: Section II describes the problem structure for the control. Section III establishes a MAS for the MG system. Section IV proposes the control strategy and furthermore verifies it in section V. Finally, section VI draws conclusions for this work.

\section{SYSTEM STRUCTURE}

In a system of multiple MGs, as shown in Fig. 1, each MG controls its facilities to achieve demand-supply balance and responds to the neighbor MGs' power demands. Within an MG, all the facilities are assumed to be controlled and monitored by its assigned MG. An MG is assumed to be composed of a photovoltaic generator, a microturbine, a battery and a load. They correspond to the physical, electric layer in the system.

When an MG cannot supply its load or absorb excess power from its generators, it requests support from neighboring MGs by sending messages. A communication net- 


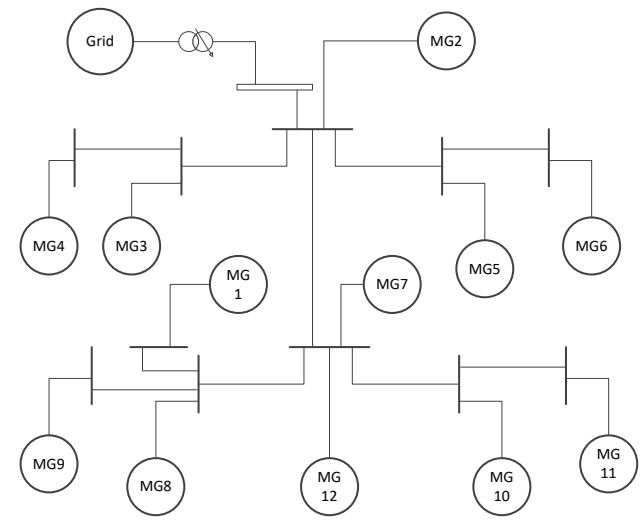

Figure 1: Studied connected MG system.

work, such as the one shown in Fig. 2, is therefore needed. In the network, an agent only communicates with others which are directly connected to it.

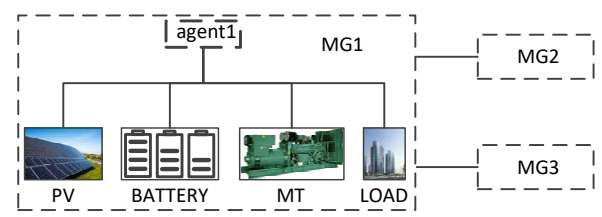

Figure 2: Part of the communication network for the studied system.

\section{MICROGRID RESOURCES CONTROL}

Each MG controls its own power sources to try to ensure its power balance. It can then operate while connected or isolated from the grid. Within one MG, the generators supply power to the load with a priority to reduce the cost. Renewable generators have the highest priority as they are fuel-free and environmentally efficient. Then energy storage systems are used to discharge when needed, or charge from local generation. Microturbines come last, as a form of last resort power source, with high fuel costs and emissions.

For stability reasons, ensuring local MG power balance is given a higher priority than responding to other MGs for cooperation. For example, when local generators cannot supply enough power to the load in the same MG, this MG requests power from its neighbors, and does not answer to other MGs' requests. If its supply-demand balance is guaranteed using local resources, it responds to other MGs' power requests based on its output flexibility.

This flexibility is defined by (1)-(3), in which all variables are associated with the $i^{\text {th }}$ MG. The output power of an MG is counted as positive when power is exported to the network. $p_{i}^{c a p, \text { min }}$ is the minimal power an MG can exchange with the network (for example, due to its renewable generation being higher than its load) and $p_{i}^{c a p, \max }$ is the maximal power. These equations are directly derived from the generators and storage systems behaviors.

$$
\begin{aligned}
& p_{i}^{\text {cap }, \min }=p_{P V, i}+p_{M T, i}-\left|p_{E S S, c h, i}^{\max }\right|-p_{\text {load }, i} \\
& p_{i}^{\text {cap }, \max }=p_{P V, i}+p_{M T, i}-\left|p_{E S S, d c h, i}^{\max }\right|-p_{\text {load }, i}
\end{aligned}
$$

$$
\left|p_{i}^{\text {dem }}\right|=\min \left(\left|p_{i}^{c a p, \min }\right|,\left|p_{i}^{c a p, \max }\right|\right)
$$

Three cases are then distinguished, depending on the values of $p_{i}^{c a p, \min }$ and $p_{i}^{c a p, \max }$ :

- If both are positive, the MG has excess power and requests neighbor MGs to absorb power.

- If both are negative, the MG lacks power and request neighbor MGs to supply power. The MG thus generates power requests according to (3).

- The last case is if one is negative and the other positive, then the MG can either absorb or output power to neighboring MGs. Such MGs act as responders to other MGs' requests.

Note that the power values in (1)-(3) can be either forecasts or measurements. In the following, we assume these are forecasts, i.e., the algorithm is run in ahead of real-time. The maximum charge and discharge power of storage units is also limited by their state-of-charge, i.e., a full battery cannot further charge.

\section{COORDINATED MICROGRID NETWORK CONTROL STRATEGY}

In the following, the coordination strategy used for coordinating power exchanges among MGs is introduced.

\subsection{Problem Formulation}

As described above, the operation of resources inside each MG determines its coordination behavior: requester or responder. To maximize the supplied load and the efficiency of generated power in the network, coordination control is adopted for power exchanges among MGs. In this work, we focus on active power exchanges and neglect reactive power, as its impact on active power flows is limited. The integration of reactive power and voltage control are left for future work.

The cost for each requester (i.e., an MG that makes a power request to a neighbor $\mathrm{MG}$ ) is to be minimized to optimize its profit, as in (4):

$$
\min \sum_{j \in M_{i}} p_{i, j} \pi_{i, j}
$$

As an MG adjusts its output according to the system conditions, once the output of the MG is determined based on the requester's schedule, the power flow is computed, as described in section 4 . To keep the demand-supply balance in the whole network, the output of MGs is limited by (5). The sum of exchanged power of all MGs in the network is shown in the left side, where the output power can be negative or positive. This sum should be equal to the total network line losses shown on the right.

$$
\sum_{i \in T} p_{i}=\sum_{i \in T} \sum_{j \in M_{i}} p_{l_{i j}}^{\text {flow }} r_{l_{i j}}
$$

Additionally, to ensure the secure operation for the system, there are some limitations, as shown in (6) which specifies that the power flowing on a line should not exceed its capacity:

$$
p_{l_{i j}}^{\text {flow }} \leq p_{c a p, l_{i j}}
$$


Each MG has two activities when cooperating with other MGs. On the one hand, it can compensate the lack of power or absorb the excess power of its neighbors. On the other hand, it sends power requests to its neighbors when it cannot ensure its own self-sufficiency. These two conditions do not exist at the same time for an MG, but can co-exist at the same time for different MGs in the system.

If an MG responds to another MG's power request, the power it exchanges with the network is limited within its power capacity, as shown in (7). It implies that the sum of the power flowing on all lines connected to the $i^{\text {th }}$ MG should be within the limitations of the MG capacity. For example, in Fig. 1, the sum of $p_{21}, p_{23}$, and $p_{24}$ (the flows on the lines connectings MG2 and MG1, 3 and 4, respectively) should be within MG2's power capacity noted $\left[p_{2}^{c a p, \min }, p_{2}^{c a p, \max }\right]$, from (1)-(2).

$$
p_{i}^{c a p, \min } \leq \sum_{j \in M_{i}} p_{l_{i j}} \leq p_{i}^{c a p, \max }
$$

\subsection{Coordination Control Method}

An overview of the proposed control system is shown in Fig. 3. An MG can be a requester under power imbalance or a responder with assisting neighbor requester. To schedule the exchanged power with each of its neighbor MGs, a given MG that sends out a power requests receives in return the power capacity of the neighbor MGs. To keep the power flows within line capacity limits, the output power of each MG in the network is used to calculate the power flow. The information transfered among the MGs corresponds to the power capacity of all neighbor MGs $p_{i}^{\text {cap, min }}$ and $p_{i}^{\text {cap,max }}$ for power dispatching.

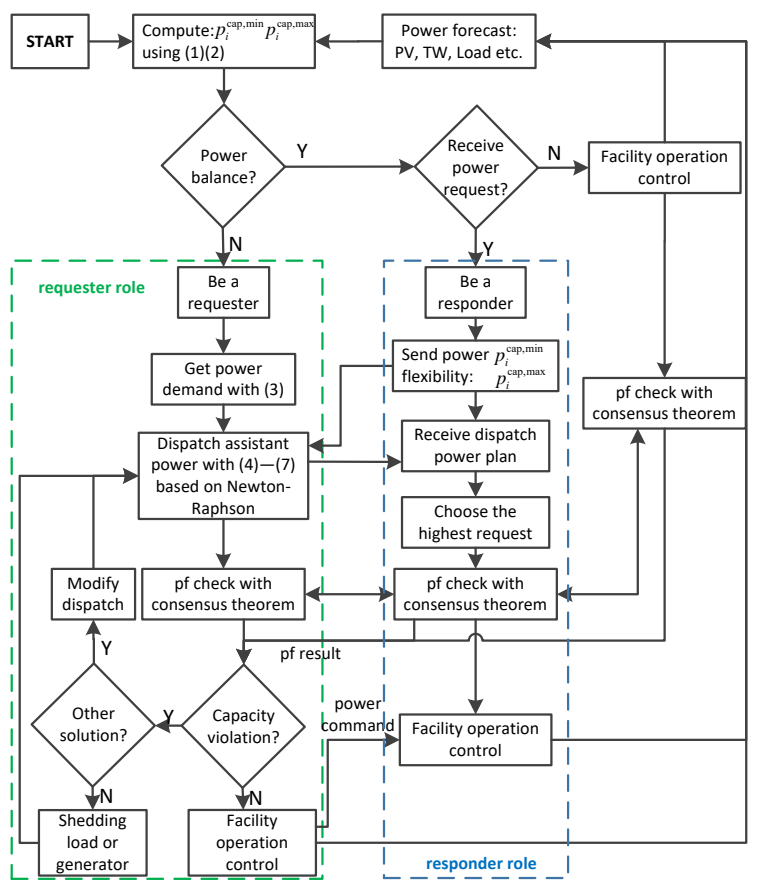

Figure 3: Control strategy flowchart within an MG.

Combining these information, the optimization problem described in (4)-(7) is solved. The Newton-Raphson method [AbbasbandyAbbasbandy2003], which is commonly used for such problems, is adopted to optimize dispatching in the system based on (4)-(5). To guarantee against the line capacity violation in (7), a distributed power flow $(\mathrm{PF})$ calculation with consensus theorem is adopted. Comparing the line capacity with line power, results show the security of schedule. The procedure of PF check will be discussed in detail in the following section. If there is a secure solution, the scheduling process finishes, and the next scheduling step within the MG is run. Otherwise, the schedule fails. If no feasible schedule is obtained, this means that some constraints cannot be met, and that the stability of the system cannot be ensured. To guarantee the secure operation of the grid, the MG sheds load or curtails renewable power generation.Each responder provides assistance to one requester for each coordination cycle. If it receives multiple requests at the same time, a priority order is used, where the largest requests are answered first, until the responder's response capacity is reached. The requester then implements the dispatch resulting from the new exchanges.

Fig. 4 shows an example of messages exchanged during the negotiation process between two agents, during the coordination. The system used in the example is shown in Fig. 1. Based on the facility power forecasts, if MG3 is lacking power or has excess power, it schedules a power exchange with its neighbors. Information on the flexibility of the responder is sent to the requester for power dispatching with the Newton Raphson method. Requester MG3 then sends to corresponding exchange schedule to responder MG2. Based on this the power flow is calculated in each agent with the consensus theorem. Line capacity constraints are further checked and the results determine whether the schedule is feasible. If no constraint is violated, the schedule is implemented and a new control cycle starts. Otherwise, MG3 redipatches power and the coordination schedule is checked again.

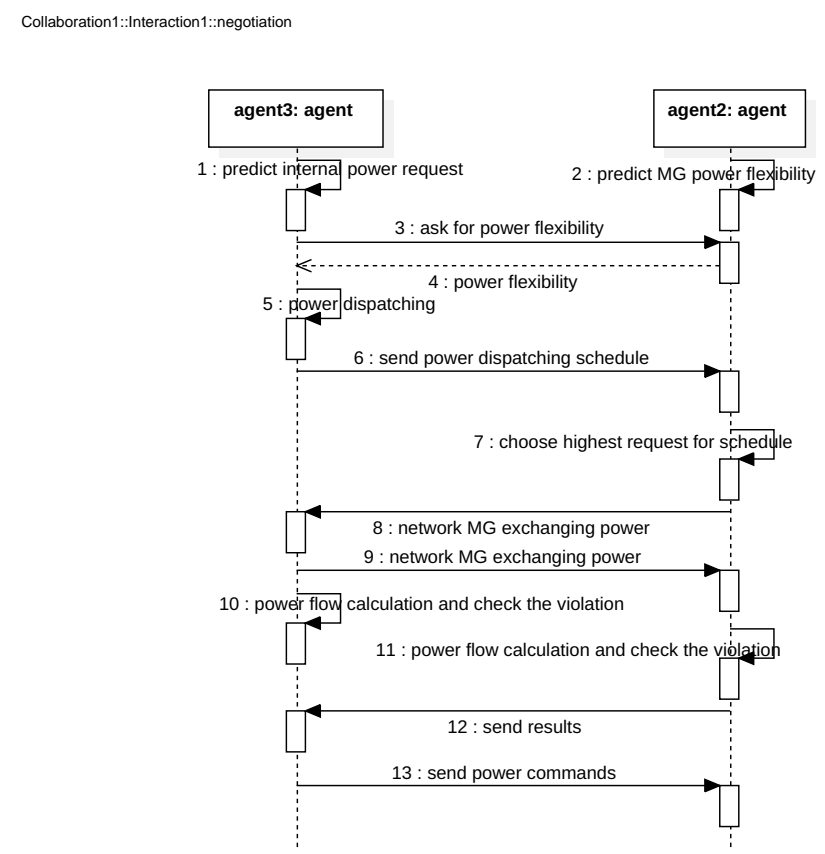

Figure 4: Coordination process example among agents. 


\subsection{Power Flow Algorithm}

In the studied networked MGs system, it is assumed that the length of the lines between MGs is limited and that their resistance cannot be neglected. An improved DC power flow that considers line resistance (instead of reactance) is thus adopted. The loss on a line $p_{l_{i j}}^{\text {loss }}$ is shown in (8). This loss power is equivalent to including loads at the terminal MGs as shown in (8)-(10) [Hongfu, Xianghong, Zhiqiang, Chong, Hao, and ShixiaHongfu et al.2014].

$$
\begin{gathered}
p_{l_{i j}}^{\text {loss }}=\left(p_{l_{i j}}^{\text {flow }}\right)^{2} r_{l_{i j}} \\
p_{i}^{\text {equ }}=\sum_{j=1, j \in M_{i}} \frac{p_{l_{i j}}^{\text {loss }}}{2} \\
p_{i}^{\text {mod }}=p_{i}-p_{i}^{\text {equ }}
\end{gathered}
$$

The resulting improved formulation of DC power flow is shown in (11)-(15) [Stott, Jardim, and AlsacStott et al.2009]:

$$
\begin{gathered}
\delta=\left[\delta_{1}, \delta_{2} \cdots, \delta_{i}, \cdots, \delta_{n}\right]^{T} \\
B=\left[\begin{array}{ccc}
1-\sum_{j=2, j \in M_{i}} b_{1 j} & \cdots & b_{1 n} \\
\vdots & \ddots & \vdots \\
b_{n 1} & \cdots & 1-\sum_{j=1, j \in M_{i}, j \neq n} b_{n j}
\end{array}\right] \\
-P=B \delta \\
p_{l_{i j}}^{\text {flow }}=\frac{\delta_{i}-\delta_{j}}{x_{l_{i j}}} \\
P=\left[p_{1}^{\text {mod }}, p_{2}^{\text {mod }} \cdots, p_{i}^{\text {mod }}, \cdots, p_{n}^{\text {mod }}\right]^{T}
\end{gathered}
$$

\subsection{Consensus-Based Coordination}

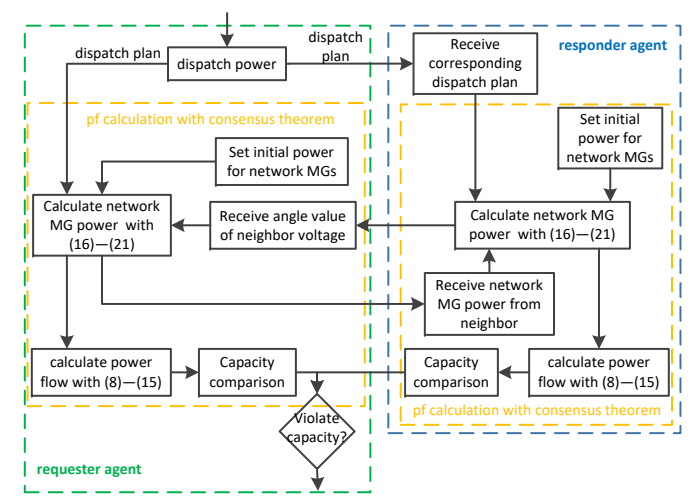

Figure 5: Control strategy flowchart for the network.

Each MG and each agent needs to receive information from other agents to decide on its actions. This information is called global information, and corresponds to the output power of all MGs. Fig. 5 shows the PF check of Fig. 4 in detail. From it, the global information is "discovered" using a consensus-based algorithm described in (16)-(21) [Xu and LiuXu and Liu2011], where each agent analyzes the information (the exchanged power $p_{i}$ of all the MGs in the network) received from neighbor agents to derive its own local information. The use of this method helps reduce single points of failure in the system, in the hope to further reduce the impact of any type of failure.

Equation (16) is the formulation of the ith microgrid when updating the global information using the neighbors' information, $p_{i}$ is the exchanged power of each MG in the network:

$$
p_{i}^{k+1}=p_{i}^{k}+\sum_{j \in N} a_{i j}\left(p_{j}^{k}-p_{i}^{k}\right)
$$

Based on (16), the whole system information is discovered by (17), where $I$ is the identity matrix:

$$
\begin{gathered}
P^{k+1}=(I+A) P^{k}=D P^{k} \\
A=\left[\begin{array}{ccc}
-\sum_{j \in M_{i}} a_{1 j} & \cdots & a_{1 n} \\
\vdots & \ddots & \vdots \\
a_{n 1} & \cdots & -\sum_{j \in N} a_{n j}
\end{array}\right] \\
D=\left[\begin{array}{ccc}
1-\sum_{j \in M_{i}} a_{1 j} & \cdots & a_{1 n} \\
\vdots & \ddots & \vdots \\
a_{n 1} & \cdots & 1-\sum_{j \in N} a_{n j}
\end{array}\right]
\end{gathered}
$$

To make this approach stable, $a_{i j}$, the element in $A$ is defined in (21) [Xu and LiuXu and Liu2011]:

$$
a_{i j}= \begin{cases}\frac{1}{\sum_{M_{i}} 1+\sum_{M_{j}} 1}, & \text { if } j \in M_{i} \\ 1-\sum_{M_{j}} \frac{1}{\sum_{M_{i}} 1+\sum_{M_{j}} 1}, & \text { if } j=i \\ 0, & \text { otherwise }\end{cases}
$$

\section{SIMULATION RESULTS}

The proposed control strategy is tested on the IEEE 13node-based system shown in Fig. 1. Line parameters are from [KerstingKersting2001]. In the simulation, the battery in MG3 is assumed to be disconnected in the whole day, e.g., for maintenance. The relationship between cost and amount of electricity generation in MG $i$ is assumed to be defined as a quadratic function, as in (22). Each responder sends the cost to the requester. The sum of the costs for buying electricity from responders is minimized as the profit of requester is maximized, as shown in (23). This equation can then replace (4) in the Newton Raphson method. Table 1 shows the cost parameters that were arbitrarily selected for this study for the responder MGs.

$$
\begin{gathered}
Y_{i}=B_{i} p_{i}+C_{i} p_{i}^{2} \\
\min \sum_{j \in M_{i}} Y_{j}
\end{gathered}
$$

Two scenarios are considered. In a reference scenario (without coordination), we use a selfish, self-sufficient control strategy, where MGs do not coordinate with each other. 
Table 1: Price parameters of the electricity in each MG.

\begin{tabular}{|l|c|c|c|}
\hline MG & MG3 & MG2 & MG4 \\
\hline$B_{i}$ & 1 & 3.5 & 6.3 \\
\hline$C_{i}$ & 0.006 & 0.004 & 0.009 \\
\hline
\end{tabular}

If demand and supply of an MG are not balanced, the MG sheds loads with the lowest priority or curtails power generation to try to reach power balance. In the second scenario (with coordination), the method proposed in this paper is used and evaluated. To compare results for both scenarios, the shed load, the curtailed generation power, and costs are used to represent the performance of the control.

For the reference scenario, internal control results for MG3 (which includes no storage) are shown in Fig. 6. The output of each component is shown in (a), the lack of power is shown in (b). As there is no MG to absorb the excess power or supply the load, load and generation shedding are used.

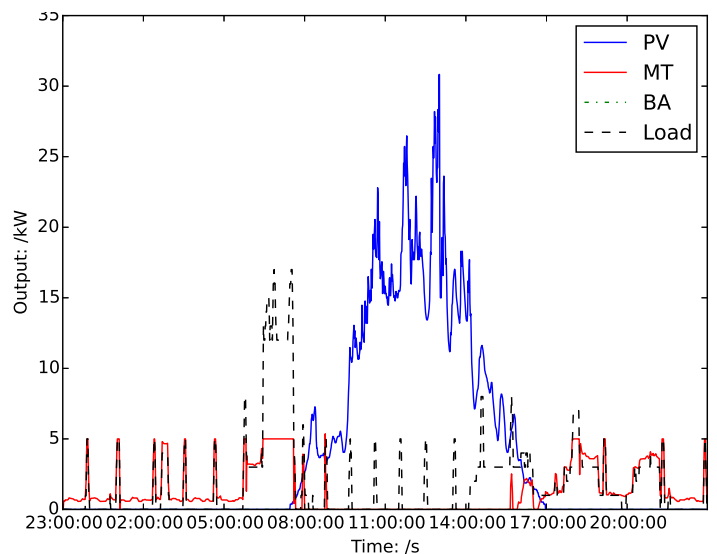

(a) Local resources output

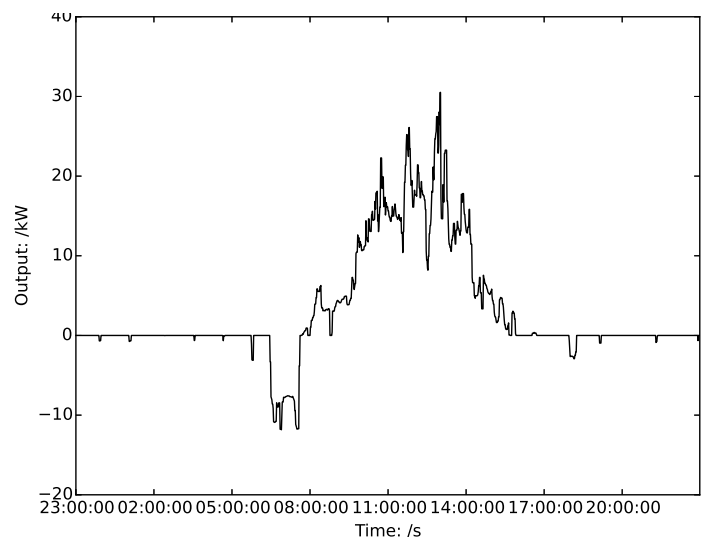

(b) Power deficiency

Figure 6: Behavior of MG3.

With coordinated control, the network operates as a storage unit to eliminate MG power imbalances, as shown in Fig. 7. Coordination is thus achieved among MGs. The power output of MG2, 3 and 4 is shown in (a). As a consequence of the imbalance in MG3 (which is unable to store its excess PV generation and supply the suplus load), its neighbors, i.e., MG2 and MG4, provide the necessary power assistant. Due to (6), the sum of the power flowing from MG2 and MG4 to MG3 is equal to the value of the MG3 request. Other MGs do not participate in the coordination, so their output is not shown.

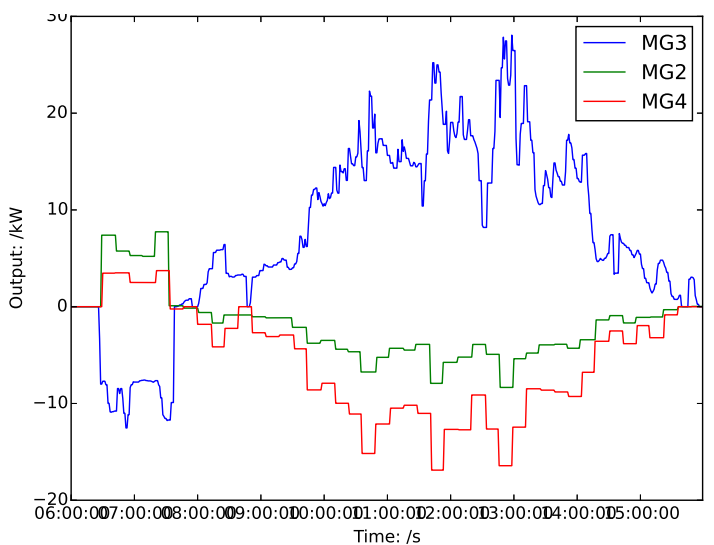

(a) MG exchanged power

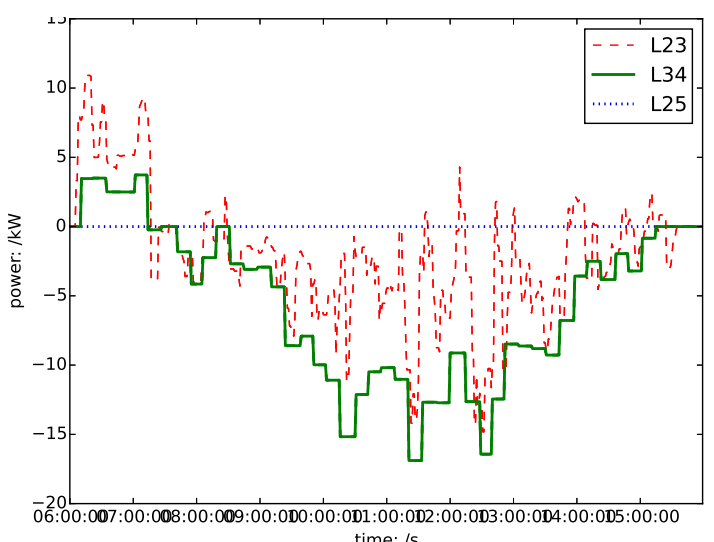

(b) Line power flows

Figure 7: Coordination results for MG3.

Table 2: Simulation results.

\begin{tabular}{|c|c|c|c|c|c|c|c|c|}
\hline & \multicolumn{2}{|c|}{ SL $(\mathrm{kWh})$} & \multicolumn{2}{c|}{ CG (kWh) } & \multicolumn{2}{c|}{ TC (EUR) } & \multicolumn{2}{|c|}{ EG (kWh) } \\
\hline CO & 0 & 1 & 0 & 1 & 0 & 1 & 0 & 1 \\
\hline MG3 & 11.77 & 0 & 85.28 & 0 & 0 & -16.74 & 0 & 97.05 \\
\hline MG2 & 0 & 0 & 0 & 0 & 0 & 3.834 & 0 & 33.17 \\
\hline MG4 & 0 & 0 & 0 & 0 & 0 & -20.57 & 0 & 61.97 \\
\hline
\end{tabular}

Numerical results are summarized in Table 2, which includes the shed load (SL), the curtailed generation (CG), the trading cost (TC) and the exchanged power (EG) with the network, from the point-of-view of MG3. Results without (0) and with (1) coordination (CO) are compared. Without coordination, MG3 sheds $11.77 \mathrm{kWh}$ of load and $85.28 \mathrm{kWh}$ of generation over the day, as there is no power exchange possibility between MG3 and the network. The corresponding exchange cost is obviously zero. For the control with coordination, load shedding and curtailments are avoided. As the excess power of MG3 is absorbed by neighbor MGs, MG3 can thus generate a profit (i.e., a negative cost). The coordination between MGs helps solve the power imbalance. 
This improves the system resilience and maximize the use of renewable energy. As the power demand from MG3 is satisfied by its local resources and the other MGs, the coordination control strategy achieves its objective.

A deeper study on MG coordination with distributed MAS will focus on the comparison with the central method which is lacking of flexibility on MG scalibility.

\section{CONCLUSIONS}

This paper has proposed a strategy for coordinating power flows connected MGs, so they can exchange power when necessary. The proposed approach combines consensus, power flow and dispatching algorithms to achieve coordination of the different agents. Preliminary results of simulations with an IEEE 13 node network show that this strategy is feasible, and can help reduce costs and improve overall resilience. Future work will focus on improving the coordination strategy, with a focus on further distributing the control method and enhancing the pricing strategy as well as line capacity constraints handling solutions.

\section{REFERENCES}

[AbbasbandyAbbasbandy2003] S. Abbasbandy. 2003. Improving NewtonâĂŞRaphson method for nonlinear equations by modified Adomian decomposition method. Appl. Math. Comput. 145, 2âAS33 (2003), 887 - 893. https://doi.org/10.1016/S0096-3003(03)00282-0

[Asimakopoulou, Dimeas, and HatziargyriouAsimakopoulou et al.2013 G. E. Asimakopoulou, A. L. Dimeas, and N. D. Hatziargyriou. 2013. Leader-Follower Strategies for Energy Management of Multi-Microgrids. IEEE Transactions on Smart Grid 4, 4 (Dec 2013), 1909-1916. https://doi.org/10.1109/TSG.2013.2256941

[Fathi and BevraniFathi and Bevrani2013] M. Fathi and H. Bevrani. 2013. Adaptive Energy Consumption Scheduling for Connected Microgrids Under Demand Uncertainty. IEEE Transactions on Power Delivery 28, 3 (July 2013), 1576-1583. https://doi.org/10.1109/TPWRD.2013.2257877 W. Hongfu, T. Xianghong, Z. Zhiqiang, G. Chong, Y.
Wianghong, Zhiqiang, Chong, Hao, and ShixiaHo Hao, and M. Shixia. 2014. An improved DC power flow algorithm with consideration of network loss. In Power System Technology (POWERCON), 2014 International Conference on. 455-460. https://doi.org/10.1109/POWERCON.2014.6993704

[Katiraei, Iravani, and LehnKatiraei et al.2005] F. Katiraei, M. R. Iravani, and P. W. Lehn. 2005. Micro-grid autonomous operation during and subsequent to islanding process. IEEE Transactions on Power Delivery 20, 1 (Jan 2005), 248-257. https://doi.org/10.1109/TPWRD.2004.835051

[KerstingKersting2001] W. H. Kersting. 2001. Radial distribution test feeders. In 2001 IEEE Power Engineering Society Winter Meeting. Conference Proceedings (Cat. No.01CH37194), Vol. 2. 908-912 vol.2. https://doi.org/10.1109/PESW.2001.916993

[Liu, Gao, Liu, Ma, Chen, and YangLiu et al.2015] Youbo Liu, Hongjun Gao, Junyong Liu, Zhao Ma, Jinxiang Chen, and Yang Yang. 2015. Multi-agent based hierarchical power scheduling strategy for active distribution network. In Smart Electric Distribution Systems and Technologies (EDST), 2015 International Symposium on. 151-158. https://doi.org/10.1109/SEDST.2015.7315199

[Marvasti, Fu, DorMohammadi, and Rais-RohaniMarvasti et al.2014] A. K. Marvasti, Y. Fu, S. DorMohammadi, and M. Rais-Rohani. 2014. Optimal Operation of Active Distribution Grids: A System of Systems Framework. IEEE Transactions on Smart Grid 5, 3 (May 2014), 1228-1237. https://doi.org/10.1109/TSG.2013.2282867

[Nunna and DoollaNunna and Doolla2012] H. S. V. S. K. Nunna and S. Doolla. 2012. Demand Response in Smart Distribution System With Multiple Microgrids. IEEE Transactions on Smart Grid 3, 4 (Dec 2012), 1641-1649. https://doi.org/10.1109/TSG.2012.2208658

[Panteli, Trakas, Mancarella, and HatziargyriouPanteli et al.2016] M. Panteli, D. N. Trakas, P. Mancarella, and N. D. Hatziargyriou. 2016. Boosting the Power Grid Resilience to Extreme Weather Events Using Defensive Islanding. IEEE Transactions on Smart Grid PP, 99 (2016), 1-10. https://doi.org/10.1109/TSG.2016.2535228

[Roche, Blunier, Miraoui, Hilaire, and KoukamRoche et al.2010] R. Roche, B. Blunier, A. Miraoui, V. Hilaire, and A. Koukam. 2010. Multi-agent systems for grid energy management: A short review. In IECON 2010 - 36th Annual Conference on IEEE Industrial Electronics Society. 3341-3346. https://doi.org/10.1109/IECON.2010.5675295

[Stott, Jardim, and AlsacStott et al.2009] B. Stott, J. Jardim, and O. Alsac. 2009. DC Power Flow Revisited. IEEE Transactions on Power Systems 24, 3 (Aug 2009), 1290-1300. https://doi.org/10.1109/TPWRS.2009.2021235

[Wang, Chen, Wang, Begovic, and ChenWang et al.2015] Z. Wang, B. Chen, J. Wang, M. M. Begovic, and C. Chen. 2015. Coordinated Energy Management of Networked Microgrids in Distribution Systems. IEEE Transactions on Smart Grid 6, 1 (Jan 2015), 45-53. hittps://doi.org/10.1109/TSG.2014.2329846

[Wang, Chen, Wang, and ChenWang et al.2016] Z. Wang, B. Chen, J. Wang, and C. Chen. 2016. Networked Microgrids for Self-Healing Power Systems. IEEE Transactions on Smart Grid 7, 1 (Jan 2016), 310-319. https://doi.org/10.1109/TSG.2015.2427513

[Wu and GuanWu and Guan2013] J. Wu and X. Guan. 2013. Coordinated Multi-Microgrids Optimal Control Algorithm for Smart Distribution Management System. IEEE Transactions on Smart Grid 4, 4 (Dec 2013), 2174-2181. https://doi.org/10.1109/TSG.2013.2269481

[Xu and LiuXu and Liu2011] Y. Xu and W. Liu. 2011. Novel Multiagent Based Load Restoration Algorithm for Microgrids. IEEE Transactions on Smart Grid 2, 1 (March 2011), 152-161. https://doi.org/10.1109/TSG.2010.2099675 